\title{
Theoretical and experimental investigation of backlash effects on a 2-DOF robotic balancing table
}

\author{
G. Bayar \\ Mechanical Engineering Department, Bulent Ecevit University, Zonguldak, Turkey, E-mail: gbayar@gmail.com
}

cross $^{\text {ref }}$ http://dx.doi.org/10.5755/j01.mech.23.1.14008

\section{Introduction}

Robotic balancing systems are commonly used in industry, research centers and academia. In industry, they are great helper to manufacture accurate products. For example, welding robots are used for fast and accurate production lines. They perform welding operations by following a predefined welding path/trajectory. The use of a welding robot in a production line may be inadequate for achieving high-quality welding due to that robot may have range problems. In other words, it cannot easily reach all the points which have to be welded. In order to overcome such problems, operation algorithms of a welding robot are connected to that of a robotic balancing platform. Both robotic systems are used together so that all the problematic points and locations become accessible.

In research centers, robotic balancing systems are used to test the methodologies like mathematical methods, system models and controllers. Such systems give the researchers opportunities to verify their new approaches. Use of them provides a facility tool to develop new prototypes and see their working performances.

In engineering faculties and student laboratories of universities, robotic balancing tables are commonly used to show the working principles of a 2-DOF robotic system. They are also proper educational tools to illustrate the meaning of control strategies. Furthermore, such robotic systems make them easy to understand the issues of performance evaluation of different models accompanied with different controllers for a real system.

In this study, backlash effects on a 2-DOF robotic balancing table are investigated to provide feedbacks for the developers to create more accurate systems. Backlash phenomena are defined by following the descriptions presented in literature and modeled for the system introduced in this study. The equations of motion principles of the 2DOF robotic system are also constructed. To test the procedures introduced, motion of a ball on the balancing table is taken into account. The proposed backlash observation system, the motion model of the balancing table and the ball's movement are interconnected into a single system to see the backlash effects on the system performance. System is controlled to be able to locate the ball at the center of the balancing table. To achieve this control objective, a PID control strategy is coupled with the process. The proposed system is simulated first then it is adapted into an experimental setup consisting of two driving units. The setup includes also an encoder input card and a motion control unit. The feedback information, the location of the ball on the balancing table, is obtained using the data coming from a top-head camera.

\section{Literature review}

Literature studies can be reviewed into two main groups. The first group is about the development of robotic balancing systems which are commonly used for the industrial applications and research activities. The second group is related to the investigations of backlash problem in the mechanical systems.

In [1], the balancing performance of an automatic ball balancing system without fluid lubrication is studied. The effects of dry friction on the working performance of a balancing table are investigated. It is stated that without using fluid lubrication causes to dry friction and badly affects the motion of the system. In order to solve this problem, a system model with including dry friction phenomena is proposed. The methodology is verified using an experimental setup of a ball balancing table. In [2], a study related to investigating an automatic balancing mechanism with multi-ball is conducted. The main purpose of the study focuses on making analytical and experimental studies to show the dynamic performance of an automatic balancer that is built to suppress vibration in eccentric systems. In [3], backlash effects on a cable actuated device are investigated. The system control is combined with the backlash model and the overall effects are observed. The proposed system is tested in an experimental setup. Backlash in the system is observed in real-time and its effects on desired trajectory tracking purpose are explored. Tracking errors obtained with and without implementing the backlash model into the system are illustrated in order to make verification. In $[4,10]$, a backlash compensation for discrete time nonlinear systems is proposed. The system is developed using dynamic inversion approach offered by neural networks. The proposed algorithm observes the backlash and its effects. The control strategy created using backstepping technique provides an adaptive backlash compensation opportunity. In [5], the application of hybrid control of a balancing robotic system is studied. The effects of backlash on the system performance are investigated. In order to create an automatic robotic system, a sliding mode based controller is developed. The controller introduced in the study can be switched according to the conditions of backlash. In [6], nonlinear identification of backlash phenomena is performed for robot transmission systems. The study states that the backlash is measured by the users and / or manufacturers. The system introduced proposes an autonomous backlash observation system for the robot transmissions. It is simulated but not experimentally tested. In [7], a methodology for backlash feedforward compensation is introduced. It is adapted to a robotic system and its performance is observed. In [8], performance analysis of a cable driven flexible robotic systems 
are studied. Asymmetric backlash profiles are taken into account in order to make enhancement in performances. In [9], backlash effects on surgical robotic tasks are investigated. System performance is observed to show that backlash affects proficiency in basic surgical skills. Backlash is added to a real surgical robotic system using proper software. By this way, the proposed procedure is verified. In [11], backlash modeling and its compensation in robotic manipulators are studied. In order to measure the kinematic errors caused by harmonic drive and backlash, an experimental system is developed and used.

What distinguishes this work from the ones presented above is that the study focuses on a 2-DOF robotic balancing system plus backlash effects on motion. The position control of a ball on the balancing table is investigated with considering the backlash effects. These steps are performed in a systemic and comprehensive way. Systemic because the methodology introduced in this paper considers the expectations of end users. The study is comprehensive because the procedure constructed makes a connection through the system mathematical modeling and simulation to successful experiments with verification.

\section{Modeling of backlash}

In mechanical systems, backlash is encountered as a problem. When backlash occurs in a system, the system runs with an unaccepted / unpredicted behavior. In order to solve such a problem, backlash and its effects on the system should be mathematically modeled and fed into the system. By this way required actions can be taken before the backlash occurs.

Fig. 1 presents a system including two rotating masses. Backlash gap is indicated with $2 \alpha$. Elasticity and viscous damping parameters of the inertia free shaft is shown by $k_{s}$ and $c_{s}$. Mass moment of inertia of the motor side of the system is given by $J_{m}$ whereas $J_{L}$ indicates that of load side. Torques of motor, shaft and load are presented by $T_{m}, T_{s}, T_{L}$, respectively. Disturbance torque is shown with $T_{d}$. Angular velocities of the motor and load sides are given by $\omega_{m}$ and $\omega_{L}$, respectively.

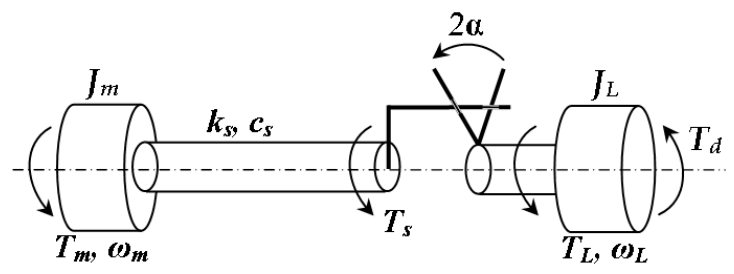

Fig. 1 Physical model of a rotating system

General torque expression for the system shown in Fig. 1 can be constructed based on [14-17]:

$$
T_{s}=k_{s} \theta_{s}+c_{s} \dot{\theta}_{s}
$$

This equation presents a nonlinear system dynamics that includes shaft twist angle and its derivative. $\theta_{s}$ indicates the shaft twist and can be defined by the following:

$$
\theta_{s}=\theta_{d}-\theta_{b}
$$

The angular difference between the motor and load that is known as twist angle is indicated by $\theta_{d}$. Back- lash angle is incorporated with the angle of $\theta_{b}$. In a general form, backlash angle is defined as:

$$
2 \theta_{b} \leq|2 \alpha| \text {. }
$$

As long as the torque value $\left(T_{s}\right)$ equals to zero, there is no contact between the teeth. In the system shown in Fig. 1, there might be three different cases; the first case is the contact at the backlash angle of $+\alpha$. In the second case, the contact occurs at the backlash angle of - $\alpha$. In the third case there is no contact. The last case can be defined with the following representation:

$$
\dot{\theta}_{d}-\dot{\theta}_{b}=\frac{-k_{s}}{c_{s}}\left(\theta_{d}-\theta_{b}\right)
$$

The solution of Eq. (4) is found as:

$$
\theta_{d}-\theta_{b}=\left(\theta_{d}-\theta_{b}\left(t_{0}\right)\right) \exp \left(\frac{-k_{s}}{c_{s}}\left(t-t_{0}\right)\right) .
$$

Once the shaft twist angle indicated by $\theta_{d}$ is provided, backlash angle can be found using Eqs. (3) and (5).

In order to model backlash phenomena, there are some approaches which are commonly used. One of these approaches $[14,15,17]$ is the exact backlash model presented in Eq. (6).

$$
\dot{\theta}_{b}=\left\{\begin{array}{cc}
\max \left(0, \dot{\theta}_{d}+\frac{k_{s}}{c_{s}}\left(\theta_{d}-\theta_{b}\right)\right), & \text { if } \theta_{b}=-\alpha ; \\
\dot{\theta}_{d}+\frac{k_{s}}{c_{s}}\left(\theta_{d}-\theta_{b}\right), & \text { if }\left|\theta_{b}\right|<\alpha ; \\
\min \left(0, \dot{\theta}_{d}+\frac{k_{s}}{c_{s}}\left(\theta_{d}-\theta_{b}\right)\right), & \text { if } \theta_{b}=+\alpha .
\end{array}\right.
$$

Another commonly used backlash model is deadzone model $[14,15,17]$. This model is constructed by doing simplification on the exact backlash model given above. In this model shaft damping effect is not taken into account. This model can be reliably used only if small or no damping occurs in the shaft. In the real time applications from which high accuracy is not expected, deadzone backlash model is mostly preferred due to its simplicity. The model offers the following representation for shaft torque:

$$
T_{s}=k_{s} \theta_{s}=k_{s} D_{\alpha}\left(\theta_{d}\right)
$$

where $D_{\alpha}$ is deadzone function and defined as follows:

$$
D_{\alpha}=\left\{\begin{array}{cl}
\left(\theta_{d}-\alpha\right), & \text { if } \theta_{d}>\alpha ; \\
0, & \text { if }\left|\theta_{d}\right| \leq \alpha \\
\left(\theta_{d}+\alpha\right), & \text { if } \theta_{d}<-\alpha
\end{array}\right.
$$

Neglecting shaft damping effect enables to use the deadzone model for backlash adaptation. By this way the parameters of the backlash model indicated by $\beta_{1}, \beta_{2}$ and $\theta_{b}$ in Fig. 2 can be estimated. The model is constructed such that shaft has no internal damping, no spring effects and no 
inertia. Following these assumptions and using an adaptation approach, an estimation procedure is constructed and the system can be linearly controlled.

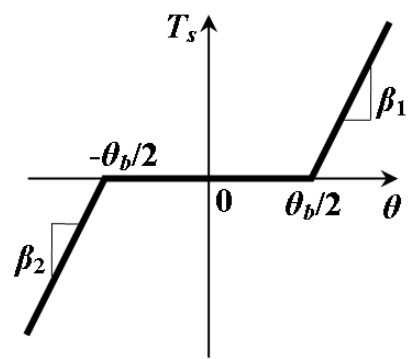

Fig. 2 Deadzone backlash model

\subsection{Development of a torque observing system}

When the system introduced in Fig. 1 is loaded, there might be an unexpected external torque effect (this can also contribute backlash). The best way to observe the effects of disturbance torque and handle the backlash in the system is to construct a torque observer and couple it with the whole system model. By this way, in addition to observing disturbance torque effects, state of the system is to be estimated.

Torque observing system is briefly presented in Fig. 3. The observing mechanism takes place in the observer block in the system. Reference input and controller are shown by $R$ and $G_{c}$. Disturbance torque effect is indicated by $T_{d}$. Torque estimator and observer are constructed based on [15]:

$$
\left.\begin{array}{l}
T_{e}=\frac{T_{m}-J_{m} \omega_{m} s}{\tau s+1} \\
\Omega=K \frac{1}{\tau s+1} T_{e},
\end{array}\right\}
$$

where system dynamics and compensator gain are shown by $K$ and $\tau$. Torque estimator $\left(T_{e}\right)$ needs motor rotational velocity and motor torque inputs. In this equation, angular acceleration is shown by $\omega_{m} s$ term which has no effects on the system in case the motor runs with constant speed. When backlash occurs in the system, the velocity is not constant anymore (this results the system accelerates).

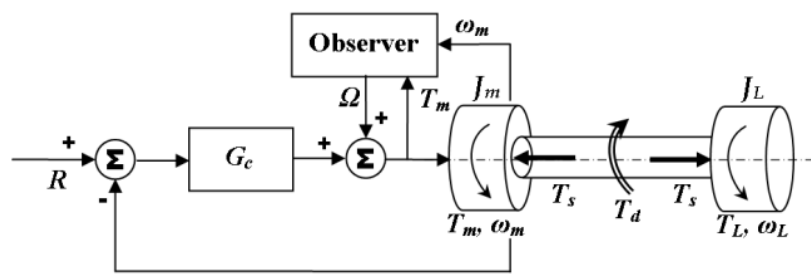

Fig. 3 Physical model including torque and backlash observing system

\section{Two-DOF robotic balancing table}

In this study, an experimental setup shown in Fig. 4 is used. It involves a robotic balancing table, and a camera attached to the top of the platform. Robotic balancer [12] is developed using two driving systems manufactured by Quanser Company (SRV02). Each driving servo unit is coupled with high resolution quadrature encoders.
The angular resolution of the encoders is 4096 pulses per revolution. Encoder data transmission, communication and encoding actions are performed using an encoder input card, manufactured by Quanser Company (Q2-USB).

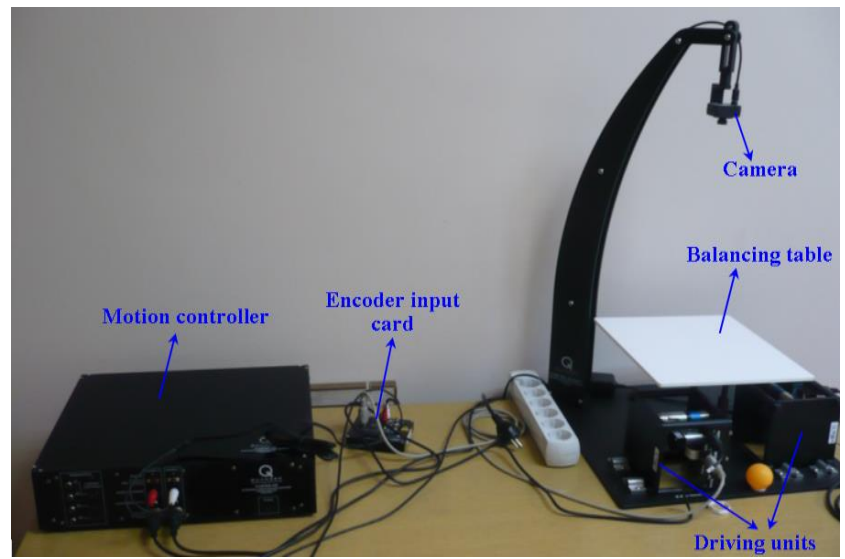

Fig. 4 Experimental setup

The balancing table can be tilted in two $(x, y)$ directions. The tilt angles are derived using both kinematics of the system and rotation information obtained from the encoders. The kinematics model of the robotic balancing system is shown in Fig. 5. Link lengths are presented by $L_{1}, L_{2}, L_{3}$ and the radius of driving unit is shown by $R_{d}$. Angular rotation and tilt angle are specified by $\theta$ and $\Phi$, respectively. The model shown in Fig. 5 represents only one DOF of the balancing system. The second DOF system is totally identical with this one. Note that the second tilt angle is obtained using the information coming from the other encoder attached to the second driving unit.

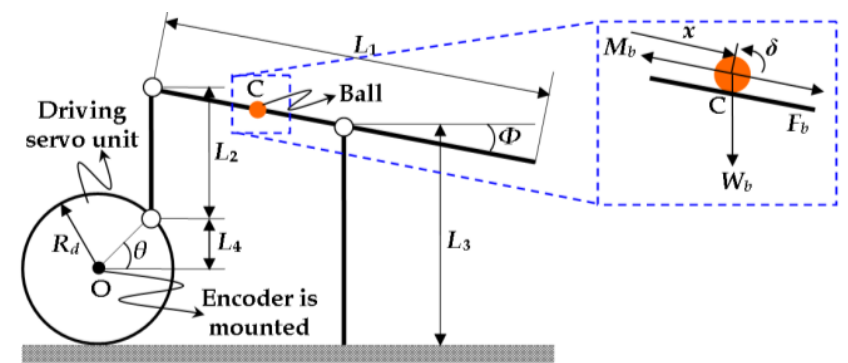

Fig. 5 Kinematic model of the system

As explained above, the performance of the balancing system under the backlash effects are tested with controlling the position of a ball. The system is controlled so that the ball should be positioned at the center of the balancing table. While achieving this objective, the effects of backlash on motion are observed. Considering the balancing system (Fig. 5-left) and the ball (Fig. 5-right) together, equation of motion of the system can be built in the following order (Eqs. 10 through 15) [13]:

$$
m_{\text {ball }} a_{\text {ball }}=F_{\text {sum }}=F_{I}+F_{G} .
$$

Forces caused from the inertia of ball and gravitational acceleration are indicated by $F_{I}$ and $F_{G}$, respectively. Mass and acceleration of the ball are specified by $m_{\text {ball }}$ and $a_{\text {ball }}$, respectively. The forces caused by gravity in $x$ and $y$ directions can be written as:

$$
F_{G}=m_{\text {ball }} g \sin (\Phi) .
$$


The force created by the ball rotation is constructed as:

$$
F_{I}=\frac{J_{\text {ball }} x}{r_{\text {ball }}^{2}},
$$

where $r_{\text {ball }}$ indicates the radius of the ball used in this study. Combining the equations above yields the following relationship:

$$
\ddot{x}=\frac{m_{\text {ball }} g \sin (\Phi) r_{\text {ball }}^{2}}{m_{\text {ball }} r_{\text {ball }}^{2}+J_{\text {ball }}} .
$$

From the geometry given in Fig. 5, one can find:

$$
\sin (\Phi)=\frac{2 R_{d} \sin (\theta)}{L_{1}} .
$$

Inserting Eq. (14) into Eq. (13) gives the following equation of motion representation of the ball:

$$
\ddot{x}=\frac{2 \theta m_{\text {ball }} g R_{d} r_{\text {ball }}^{2}}{L_{1}\left(m_{\text {ball }} r_{\text {ball }}^{2}+J_{\text {ball }}\right)} .
$$

\section{Simulation studies}

To see the performance of the model constructed above, simulation studies are conducted. All the simulation and real-time experiments are performed using MatlabSimulink environment. General view of the simulation block is shown in Fig. 6. The reference inputs for the model are shown by $x_{R}$ and $y_{R}$. System and backlash models and controller are placed in the first-left block. The details of this block are also shown inside the dashed line in Fig. 6. Backlash and disturbance torque observations are achieved in this block. The position controls of two servo driving units are performed by using a PID controller. The controller outputs are converted into voltage comments inside the Position Model block. The voltage commands for the first and second driving units, shown by $V_{x}$ and $V_{y}$, respectively, are sent through the 2-DOF robotic platform (indicated by Real Robotic System in the simulation block diagram). The outputs of the control system are position values of the ball in $x$ and $y$ directions, rotation angles of the driving units, $\theta_{x}$ and $\theta_{y}$ and the angular velocities and torques of each motor, $\omega_{m}$ and $T_{m}$.

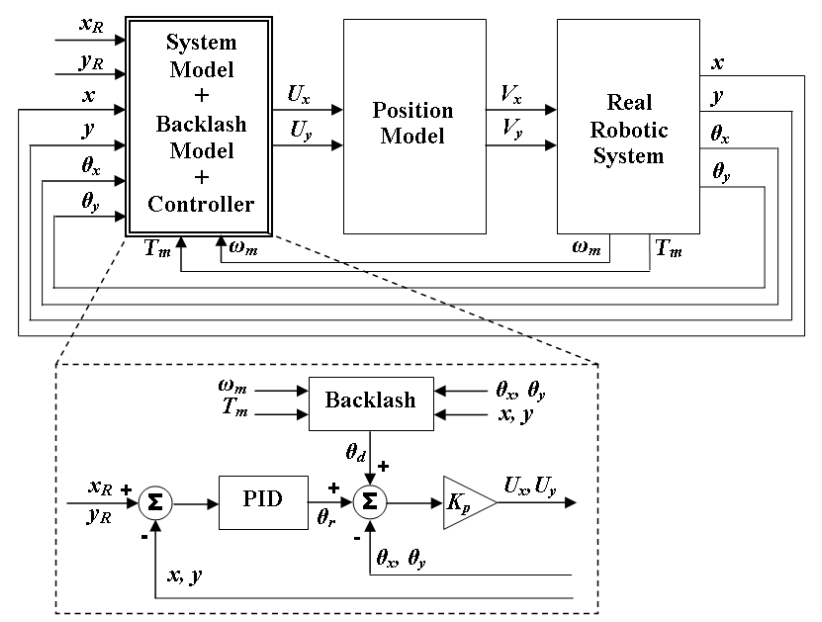

Fig. 6 Control system

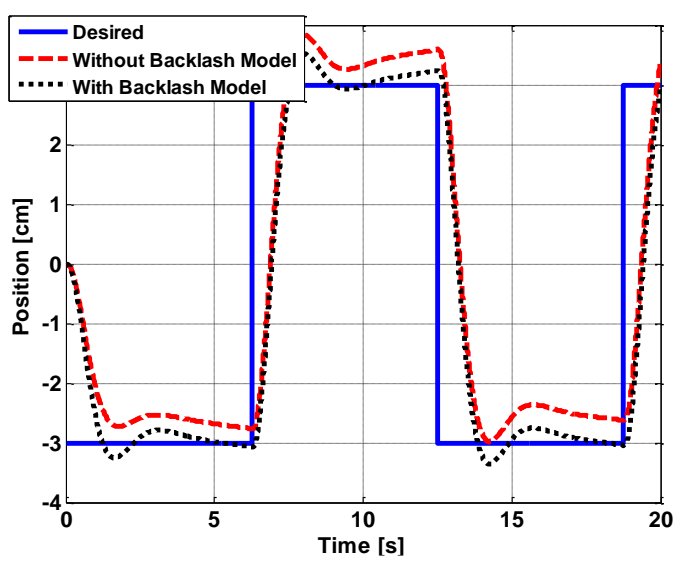

Fig. 7 Simulation results related to position control of the ball

In Fig. 8, simulation results of the rotation angles of the driving units, indicated by $\theta$ in Fig. 5, are shown. Using the same strategy, the system model is simulated with and without using the backlash observation and the results are compared with the desired reference input. The results show that the reference input is tracked with minimum tracking error when the backlash model is used.

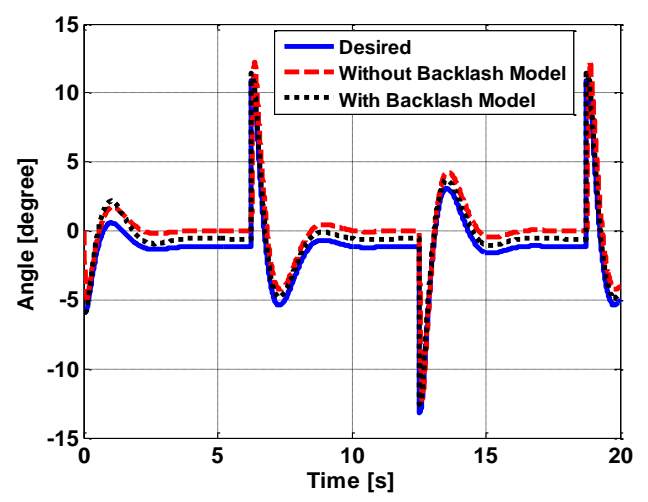

Fig. 8 Simulation results related to rotation information of the driving unit

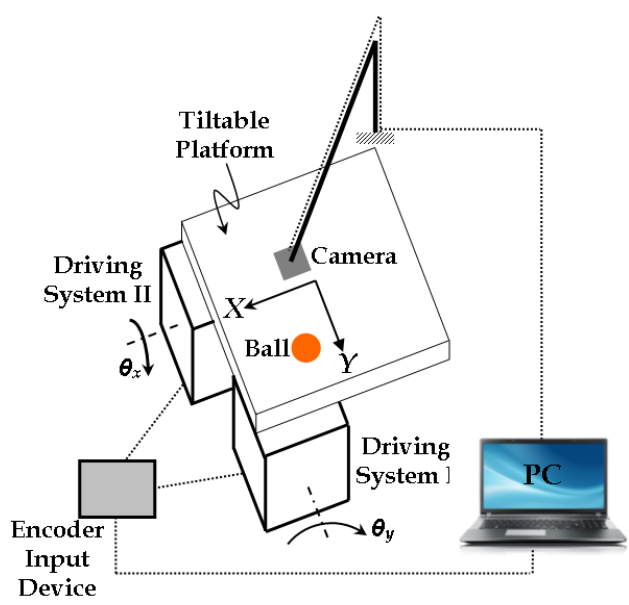

Fig. 9 Experimental setup with its details

\section{Experimental studies}

In order to verify the simulation studies, experiments are performed using the experimental setup introduced in Fig. 4. The details of the setup are also presented in Fig. 9. In this figure, driving units and mountings of 
them to the main system are illustrated. All the computational and communicational issues during the experiments are performed using Matlab/Simulink environment. The objective in these experiments is to locate the ball at the center of the tiltable platform. While controlling the balancing table to pull the ball towards the center, the effects of backlash on the system are investigated. Each experiment is conducted twice; the one is with including the backlash model into the overall system dynamics, and the other is without using it.

The first experiment results are shown in Fig. 10. The ball is placed at the left corner of the balancing table (coordinates of this points are nearly -13 and $13 \mathrm{~cm}$, in $x$ and $y$ directions, respectively). The control system efforts to get the ball at the center point of the balancing table. In this experiment, after releasing the ball free at the corner, any enforcement is not applied to it. Results obtained with and without using the backlash model are indicated by redcross and blue-dot points in Fig. 10, respectively. The dots show the ball locations during the experiments. They are obtained by using the feedback information coming from the camera. To make a comparison, centroids of the data sets are also provided. They are obtained by the use of kmeans clustering method. Note that k-means method is a data clustering approach of which purpose is to create data groups clustering around common mean vector. Details about the k-means clustering algorithm can be seen in [18].

Centroids of the data sets of two repeated experiments (with and without including backlash model) are indicated via black-crosses (Fig. 10). The desired location which shows the center point of the balancing table is also exhibited. It is shown by a green-colored-filled-circle.

As seen from the first results, when backlash model is inserted into the overall system dynamics, the ball's position is closer to the desired location. Feeding backlash information into the system model provides a positive contribution to controlling ball's position on the table.

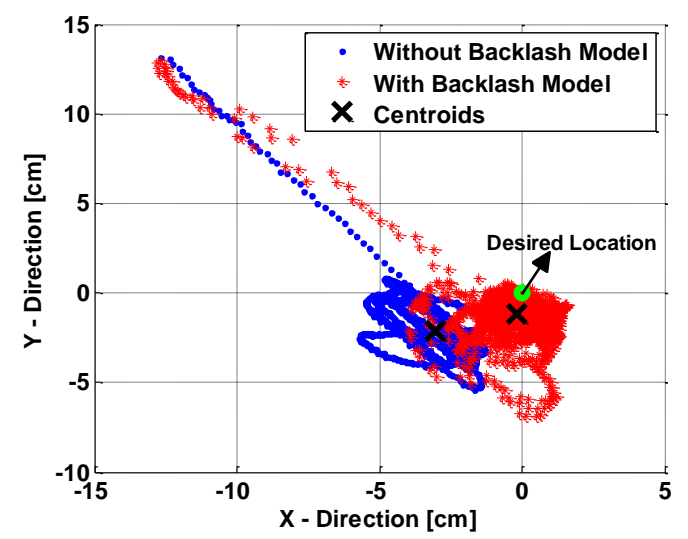

Fig. 10 Experiment results. The system proposed is run with and without including backlash model. Blackcrosses indicate the centroid of each data set

The rotation angles of the driving units are given in Fig. 11. The rotations in $x\left(\theta_{x}\right)$ and $y\left(\theta_{y}\right)$ directions are provided in Fig. 11, a and b, respectively. The desired angles of the driving units are indicated by green-dashedlined. The results obtained with and without using backlash information are shown by blue-dashed and red-solid-lines, respectively. As the desired objective, the fluctuations in the rotation angle changes should be around zero, in order to locate the ball at the center of robotic balancing table. In terms of this approach, the model including backlash information gives more reliable and stable results.

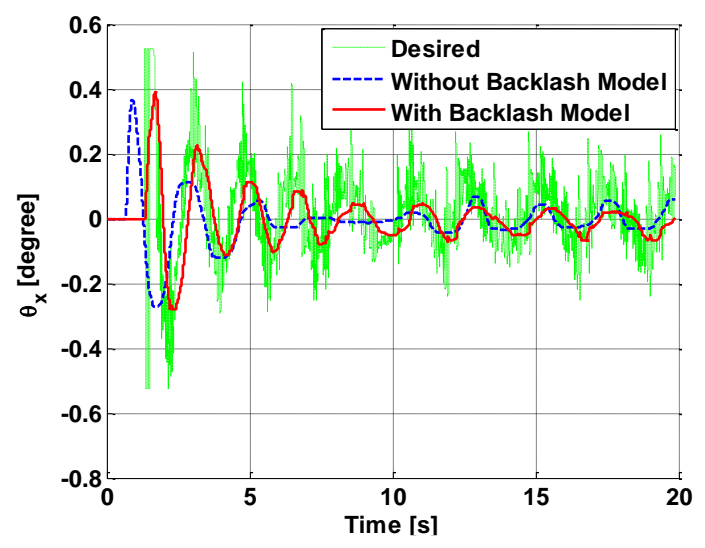

a

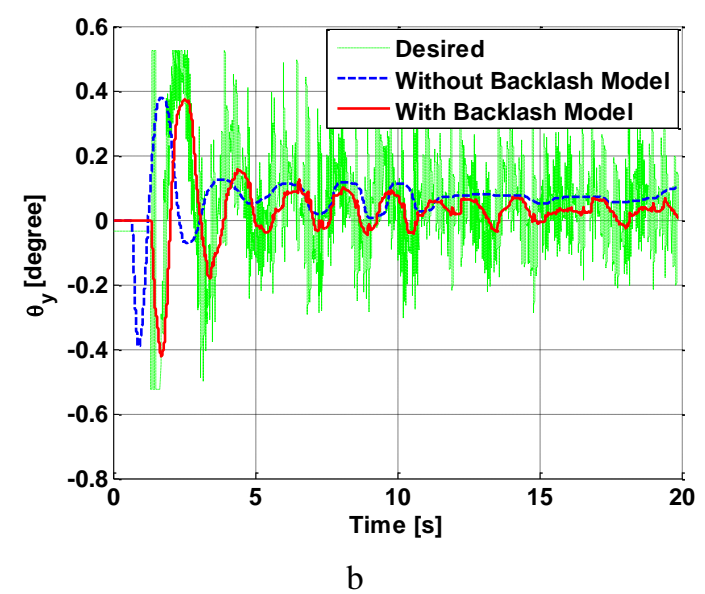

Fig. 11 Rotation angles $\left(\theta_{x}\right.$ and $\left.\theta_{y}\right)$ of driving units in $x$ (a) and $y(b)$ directions

Real-time experiments using 2-DOF robotic balancing table are conducted for two cases. As presented above, in the first case the ball is just located at the corner of the table. The ball is never enforced to pull it away from the center. In the second case, the ball is enforced to move it away from the center of the balancing table. In this case the backlash effects on the driving units, the performance of the system model and controller can be more effectively seen.

Using the same strategy introduced above, the experiments are performed with and without inserting the backlash model into the overall system dynamics.

Fig. 12 shows the results of the case where backlash model is not used. In this case whenever the ball is at the center, it is forced to move away from the center. The centroid of the data set is indicated by the red-cross. The desired location, which is the center of the table shown by the point of $(0,0)$, is given by a green-dot. To see the effects of backlash, this experiment is repeated by adding the backlash model into the system. The results are illustrated in Fig. 13. This experiment shows that the use of backlash model in the system enables to get more accurate results. The driving units and the control system work more stable to be able to locate the object on the table in a desired position. 


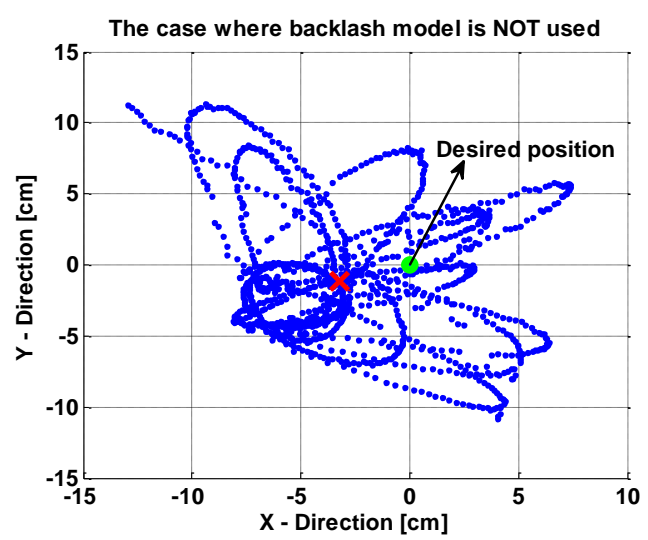

Fig. 12 Experiment results of the system (backlash model is not inserted). During the experiment, the ball is enforced to move away from the table's center. Red-cross shows the centroid of the data set

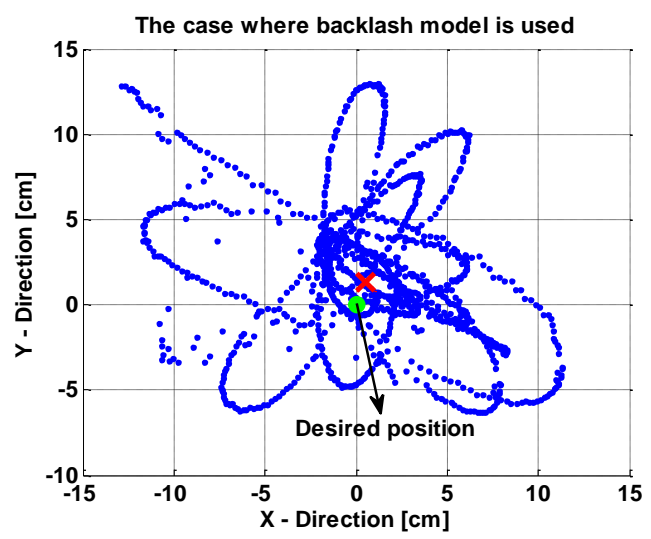

Fig. 13 Experiment results of the system including backlash model. During the experiment, the ball is enforced to move away from the table's center. Centroid of the data set and the desired location are shown by a red-cross and green-dot, respectively

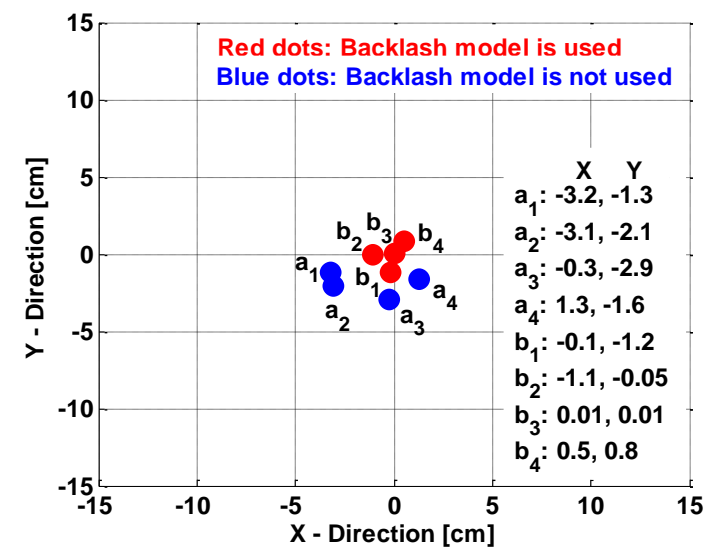

Fig. 14 Experiments conducted to show the performance and the precision positioning characteristics of the developed system when the backlash model is in use $\left(b_{i}\right)$ or not $\left(a_{i}\right)$, where $(i=1,2,3,4)$

In order to show the performance and the precision positioning characteristics of the developed system, the results of 8 experiments are presented in Fig. 14. 4 experiments are performed by using the system model including the backlash information. The other 4 experiments are conducted using the system model that does not take the backlash information into account. In Fig. 14, red dots $\left(b_{i}\right)$ indicate the ball positions in case the backlash information is used whereas blue dots $\left(a_{i}\right)$ show the ball positions when the backlash model is not used $(i=1,2,3,4)$.

\section{Analysis and conclusion}

Robotic balancing platforms are commonly used in industry and academia. For instance, in industry, welding robots can perform their jobs by the help of balancing tables because the locations, which they cannot easily access, become accessible when the balancing tables are used. In the research centers and universities, the researchers and students can see and verify the theoretical issues using robotic balancing systems. In this study, the backlash effects on a 2-DOF robotic platform are investigated. It is aimed that the performance improvement can be done by feeding the backlash information into control system. To show this purpose, position of a ball on the balancing table is controlled using a PID controller. While performing this goal, the backlash is observed and fed into the system model. In addition to doing simulations, experimental studies are conducted. The results indicate that working performance of a 2-DOF robotic balancing table can be improved if the backlash phenomena are taken into account. This can be achieved by doing that; a proper model for backlash is constructed and combined with the overall system dynamics. The methodology introduced in this study is tested for the ball position control on a 2-DOF robotic balancing table. It can be adapted to any control objective to be achieved using a 2-DOF robotic balancing system.

\section{Acknowledgement}

The author thanks to the infrastructure project of the Mechanical Engineering Department of Bulent Ecevit University (Zonguldak, Turkey) numbered 201377654622-03 for providing the 2-DOF robotic ball balancer used in this research.

\section{References}

1. Wouw, N.; Heuvel, M.N.; Nijmeijer, H.; Rooij, J.A. 2005. Performance of an automatic ball balancer with dry friction, International Journal of Bifurcation and Chaos 15(1): 65-82. http://dx.doi.org/10.1142/S0218127405012016.

2. Green, K.; Champneys, A.R.; Friswelland, M.I.; Munoz, A.M. 2008. Investigation of a multi-ball, automatic dynamic balancing mechanism for eccentric rotors, Phil. Trans. R. Soc. A 366: 705-728.

http://dx.doi.org/10.1098/rsta.2007.2123.

3. Agrawal, V.; Peine, W.J.; Yao, B.; Choi, S.W. 2010. Control of cable actuated devices using smooth backlash inverse, In: Proceedings of the IEEE International Conference on Robotics and Automation, Anchorage, Alaska, USA, 1074-1079. http://dx.doi.org/10.1109/ROBOT.2010.5509533.

4. Campos, J.; Lewis, F.L; Selmic, R. 2000. Backlash compensation in discrete time nonlinear systems using dynamic inversion by neural networks, In: Proceedings of the IEEE International Conference on Robotics and Automation (ICRA), San Francisco, CA., 1289-1295. http://dx.doi.org/10.1109/ROBOT.2000.844776.

5. Durdevic, P.; Yang, Z. 2013. Hybrid control of a two- 
wheeled automatic-balancing robot with backlash feature, In: Proceedings of the IEEE International Symposium on Safety, Security, and Rescue Robotics (SSRR), Linkoping, Sweden, 1-6. http://dx.doi.org/10.1109/SSRR.2013.6719353.

6. Hovland, G.; Hanssen, S.; Moberg, S.; Brogardh, T.; Gunnarsson, S.; Isaksson, M. 2002. Nonlinear identification of backlash in robot transmissions. In: Proceedings of the 33rd ISR (International Symposium on Robotics), Stockholm, Sweden, 1-6.

7. Mei, Z.Q; Yang, R.Q; Liang, C.; Li, G.B. 2005. The study of backlash compensation and its application in the robot checking the filter, Int. Journal Adv. Manuf. Technol 25: 396-401. http://dx.doi.org/10.1007/s00170-003-1820-x.

8. Do, T.N.; Tjahjowidodo, T.; Lau, M.W.S; Phee, S.J. 2015. Enhanced performances for cable-driven flexible robotic systems with asymmetric backlash profile, In: Proceedings of the IEEE International Conference on Technologies for Practical Robot Applications (TePRA), Woburn, MA, 1-6. http://dx.doi.org/10.1109/TePRA.2015.7219674.

9. Peine, J.W; Agrawal, V.; Peine, W.J. 2012. Effect of backlash on surgical robotic task proficiency, In: Proceedings of the IEEE International Conference on Biomedical Robotics and Biomechatronics, Roma, Italy, 799-804. http://dx.doi.org/10.1109/BioRob.2012.6290887.

10. Selmic, R.R; Lewis, F.L. 2000. Backlash compensation in nonlinear systems using dynamic inversion by neural networks, Asian Journal of Control 2(2): 76-87. http://dx.doi.org/10.1111/j.1934-6093.2000.tb00147.x.

11.Sammons, P.M; Ma.L; Embry, K.; Armstrong, L.H.; Bristow, D.A.; Landers, R.G. 2014. Modeling and compensation of backlash and harmonic driveinduced errors in robotic manipulators, In: Proceedings of the International Manufacturing Science and Engineering Conference, Detroit, Michigan; V002T02A048. http://dx.doi.org/10.1115/MSEC2014-4123.

12. Quanser 2-DOF ball balancer official web-site [accessed 23 September 2015]. Available from Internet: http://www.quanser.com/products/2dof_ball_balancer

13.2-DOF ball balancer Workbook and User Manual, Quanser Inc., 2013.

14. Nordin, M.; Galic, J.; Gutman, P. 1997. New models for backlash and gear play, Int. Journal of Adaptive Control and Signal Processing 11: 49-63. http://dx.doi.org/10.1002/(SICI)10991115(199702)11:1<49:AID-ACS394>3.0.CO;2-X.

15. Bruns, R.; Diepstraten, J.; Schuurbiers, X.; Wouters, J. 2006. Motion control of systems with backlash. Master Team Project, Technische Universiteit Eindhoven, DCT 2006.075.

16. Kalantari, R.; Foomani, M.S. 2009. Backlash nonlinearity modeling and adaptive controller design for an electromechanical power transmission system, Transaction B: Mechanical Engineering 16(6): 463-469.

17. Lagerberg, A.; Egardt, B. 2007. Backlash estimation with application to automotive powertrains, IEEE Trans. on Control Systems Technology 15(3): 483-493. http://dx.doi.org/10.1109/TCST.2007.894643.

18. Maimon, O.; Rokach, L. 2005. Clustering Methods: Data Mining and Knowledge Discovery Handbook; Springer US, 321-352. http://dx.doi.org/10.1007/978-0-387-09823-4.

G. Bayar

THEORETICAL AND EXPERIMENTAL INVESTIGATION OF BACKLASH EFFECTS ON A 2-DOF ROBOTIC BALANCING TABLE

S u m m a r y

In this study, backlash effects on a 2-DOF robotic balancing table are investigated. The study focuses on developing a backlash detecting system which consists of a backlash observer. Different backlash approaches are analyzed and a backlash modeling strategy for a 2-DOF robotic balancing table is developed. Working principles of the balancer is introduced and a detailed mathematical model is provided. Equation of motion of the system and backlash observing mechanism are combined to see the effects of backlash on motion. To see the working performance of the methodology introduced in this study, motion of a ball on the robotic balancing table is modeled with including the backlash. A simulation environment is developed to test the whole system proposed. In order to verify the results obtained in the simulation studies, an experimental setup of robotic ball balancing table is used. Position feedback of the ball is obtained via a camera attached at the top of the system. Simulation and experimental results show that better control of a robotic balancing table (thus that of ball position) can be achieved when the backlash effects are observed and fed into the system.

Keywords: 2-DOF robot, balancing table, backlash, observer, control.

Received January 20, 2016 Accepted February 06, 2017 